$\xi^{2}$

\title{
Association of Salivary Cortisol Level and Social Media Networking Usage
}

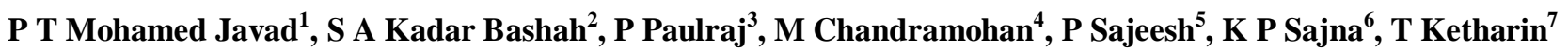 \\ Department of Biomedical Sciences, Faculty of Medicine, MAHSA University, Bandar Saujana Putra, \\ Selangor, Malaysia \\ *Corresponding author E-mail: javad@mahsa.edu.my
}

\begin{abstract}
The present study investigates the usage of social media networking behavior among the MAHSA university students by using structured questionnaire. The salivary cortisol levels among the study groups were estimated. In addition, the association between salivary cortisol and the usage of social media networking was also assessed. Saliva samples were obtained twice a day from selected subjects to analyze the cortisol level. Results of the present study indicate that the $11 \%$ of the students spend less than one hour on social media, $40.2 \%$ uses two to four hours and $46.4 \%$ more than four hours daily; whereas, $2 \%$ of the study groups are others. The cortisol levels assessed among the male and female study groups showed high cortisol concentration in both morning and evening. The cortisol levels in the morning seen among the male study group ranges from $0.13-5.49 \mu \mathrm{g} / \mathrm{dl}$ and the female cortisol level ranges from 1.11-5.72 $\mu \mathrm{g} / \mathrm{dl}$. Besides in the evening, the cortisol levels seem to be higher among the study group. The evening cortisol level of male students ranges from 0.06-4.6 $\mu \mathrm{g} / \mathrm{dl}$. Whereas in the females, cortisol level ranges $0.263 .9 \mu \mathrm{g} / \mathrm{dl}$. The average value of the cortisol concentration in the morning is seen to be higher among the female study group than the male and in the evening the male cortisol concentration is seen to be higher than the females. Results clearly demonstrated that students spending more hours in social media had higher cortisol levels when compared to less hour users. From the present preliminary study, it is clear that there is an association between salivary cortisol level and the usage of social media networking behavior.
\end{abstract}

Keywords: Cortisol; Saliva; Social Media network, Questionnaire, Stress

\section{Introduction}

Nowadays, stress is becoming a major health problem in society especially in young people. Stress is known as a state of mental or emotional state which results in adverse effects which may cause physiological and psychological tension. Stress can also affect and disrupt the human system such as the homeostasis of the body. According to Selye, stress is defined as nonspecific bodily changes that occur in response to threats or stressors [1]. There are two types of stress that normally experienced by the people which are known as the eustress and distress. Eustress is when stress triggers the function of physical or mentally such as going through a challenging work. Whereas distress is known as persistent stress that is however, when there is a chronic stress encountered, this gives a negative impact to the physiological system such as the immune function, reproduction, growth and many more [2].

When a person encounters a threat situation whether it is a physiological stress or physical stress, the Hypothalamus Pituitary Adrenal (HPA) Axis gets activated. This triggers the release hypothalamic corticotrophin-releasing hormone, Anterior Pituitary Adrenocorticotropic hormone (ACTH) and adrenal glucocorticoids which includes cortisol and corticosterone [2]. Cortisol is being used as a biomarker to test stress and related mental disease. Cortisol is a steroid hormone, which is also known as the stress hormone that can be used as an indicator for hypothalamic neural activity [3]. Moreover, cortisol also plays a crucial role in our body such as influences, regulating and modulating to changes that body encounters such as responding to threat or danger, controlling blood pressure, reduces inflammation and help in the metabolism of fat, protein carbohydrate [4]. The cortisol level may vary in the day and night by a circadian rhythm. Cortisol level will peak in the morning around 8 am and reduces by $4 \mathrm{pm}$. Any imbalance such as elevation or reduction of cortisol level can cause health problem. The morning cortisol level for males should be $0.743 \mu \mathrm{g} / \mathrm{dl}$ and female should be within 0.308 $\mu \mathrm{g} / \mathrm{dl}$. Whereas in the evening, the cortisol concentration in males should be within $1.348 \mu \mathrm{g} / \mathrm{dl}$ and females within $0.359 \mu \mathrm{g} / \mathrm{dl}$. The excess of cortisol secreted in the body can cause weight gain, blood sugar imbalances and diabetes, immune system suppression and gastrointestinal problem [5]. Whereas when there is a little amount of cortisol level can cause weight loss, muscle weakness, nausea and vomiting. Salivary cortisol is a biomarker that is used often to study the psychological stress and physical disease [6]. Besides salivary cortisol is said to be more reliable sample that can be taken for testing the stress level in a person. Although the hormone cortisol level can be obtained from the blood. Salivary cortisol is more preferable in this study due to its easy collection method, less invasive and cheaper and can be obtained from anyone.

Social media is widely used by many people as it connects people from the far destination, as a relaxing source, to obtain information and knowledge, gain new friends and many more. Although there are many advantages of the social media usage, there is a bad side too, which may cause many illnesses, lack of concentration and also stress. This is because the neuroendocrine system plays an essential role too by linking stress and ill health 
(Selye, 1976). The usage and addiction to social media networking may cause stress and elevated level of cortisol. Many from the society are still lack of knowledge towards the usage of social media in excess can cause a health problem. The main health problem which is encountered from using the social media is stress. As social media has become a port to connect with friends and family, they tend to spend more hours in social media and this eventually becomes an addiction. Besides that, because of the addiction, they even tend to stay awake for a long time to keep connect with their friend which may cause changes to their sleeping pattern. The changes in the body may also cause stress among the users. Moreover, pictures uploaded to Facebook, Instagram and other sites are to get likes and comments, when it never reaches to their expectation, this may also cause stress to the person. Other than that, some will access their social media sites in every 5 to 10 minutes to check their notification and to see anybody are online. Moreover, the researcher has stated that social media can cause depression (O'keeffe et al., 2011). In some case, the usage of social media network also leads to social bully, where pictures and text are uploaded to criticize a person, which will also disturb the state of mind of other viewers. Social networking sites are one of the major reasons to cause stress among the teens. There are only very few studies related to stress and social media networking.

Hence, the present study was carried out to investigate the association of social media networking behaviour and the stress level in MAHSA University students. The social media networking nature was assessed by administering a structured questionnaire and stress level by estimating the salivary cortisol level.

\section{Materials and Methods}

\subsection{Study Population}

Degree and diploma students of MAHSA University were used as the study population. A total of 135 students participated in the study. They were all of the age ranging from 18 to 26 .

A structured questionnaire was designed and given to the study participants to fill it online. The questionnaire included their personal information, questions related to health and usage of social media networking. The questionnaire is available on request.

\subsection{Saliva sample collection, storage and processing}

The saliva sample was collected from the study participants twice a day, one in the morning before the meal and one during the evening. The subjects were briefed on how to collect the saliva into the sample container and the quantity needed. Subjects were also instructed to not eat or drink an hour before the sample collection. This is because, the food and beverages drinks are acidic and may show the high reading of cortisol level due to the reduction of pH level (Kalman et al., 2004). Besides water also should be avoided because it may dilute the sample (Kalman et al., 2004). Once the sample container was hand over to the subjects, they were told to rinse their mouth thoroughly with water. The saliva sample collected were stored at $-20^{\circ} \mathrm{C}$.

On the day of assay, the saliva samples were brought to room temperature. Once the sample has been thawed, $1.5 \mathrm{ml}$ of the saliva was transferred to the Eppendorf tube and centrifuged at $1500 \mathrm{rpm}$ for 15 minutes to remove mucins and other unwanted matter that may interrupt with antibody binding. The supernatant was used to carry out the assay.

\subsection{Estimati9on of salivary cortisol}

The determination of salivary cortisol levels was made using the ELISA method that uses the principle of competitive binding technique. Before the procedure was done, the cortisol kit containing the reagents and microplate were brought to room temperature and was mixed vigorously before using it. $25 \mu \mathrm{L}$ of standards, control, and saliva samples were pipetted and added into the respective wells and $25 \mu \mathrm{L}$ of assay diluent was added into each Non-specific binding wells which act as a zero. Then, the enzyme conjugate that was prepared freshly was mixed thoroughly before adding into the wells. $200 \mu \mathrm{L}$ of the conjugate was pipetted and added into the respective wells. Once the enzyme conjugated was added, the plate was mixed using a plate rotator for 5 minutes at $500 \mathrm{rpm}$ and was let at room temperature for an hour to incubate. After the incubation was done, the plate was washed with $1 \mathrm{x}$ wash buffer by squirting the washing buffer into each well by pipetting $300 \mu \mathrm{L}$ of washing buffer and then discarding the liquids into the sink. This is to, wash away the freefloating molecules so that only molecules that bonds directly or indirectly are left. After washing, the plate was blotted with tissue. This plate was washed 4 times $(4 \times 300 \mu \mathrm{L})$. Then, $200 \mu \mathrm{L}$ of Tetramethylbenzidine (TMB) substrate solution was added to each well and mixed on a plate rotator. Once the mixing is done, the microplate was wrapped with an aluminium foil and was let to incubate at dark room at room temperature for 25 minutes. After incubation, the aluminium foil is removed and $50 \mu \mathrm{L}$ of stop solution was added and mixed on a plate rotator for 3 minutes at $500 \mathrm{rpm}$ until green colour turns yellow. The plate was then read by the microplate reader at $450 \mathrm{~nm}$.

\section{Results and Discussion}

Stress is being one of the psychological problems faced by many people nowadays. In this modern life, social media plays a major role in everybody's life with no age boundaries. Many people use social media as a relaxation purpose, to find friends and to pass time, but many of them failed to realize that addiction and excess usage of social media networking site can cause stress. Many studies have been done to investigate the cause of stress and to evaluate the cortisol level among the public with diseases but only a few studies were done based on social media related to stress. Thus, this current study was conducted to identify the association with salivary cortisol and usage of social media networking. From the study done, the usage of social media networking sites shows a relation between the salivary cortisol collected from the selected study group. This is because there is a close association with the cortisol level of the study group and the usage of social media networking behaviour.

\section{Social networking behaviour}

Based on the questionnaire survey conducted, $69 \%$ of the students were chosen to analyse further on their usage of social media networking behaviour. They were analysed based on selected questionnaire such as on the time spent on social media networking, addiction towards social media, usage of social media cause stress, staying up or getting up early to spend time on social media, frustration or anger when a social media networking sites goes down or unavailable and the influence of social media networking. These questionnaires was picked and analysed to study the group behaviour towards the usage of social media networking and to correlate with their saliva cortisol level.

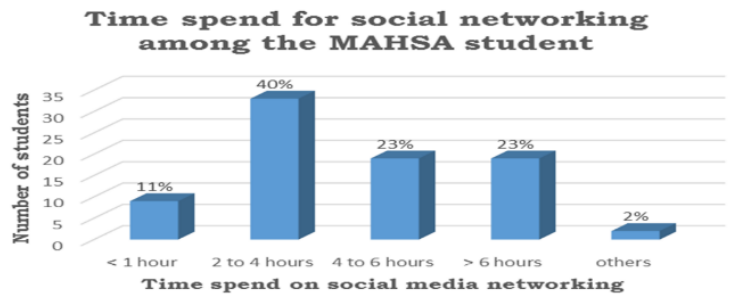

Figure 1: Time spent for social networking among the MAHSA student 
Figure 1, shows the time spend on social media networking by the study group. It was found that $40 \%$ of the study group spend two to four hours daily on social media networking, whereas $23 \%$ seen equally in those study group that spends time from 4 to 6 hours and more than 6 hours daily. $11 \%$ was seen in that study group that uses less than one hour and $2 \%$ on others. Another questionnaire was also analyzed to support their social media networking behavior as mentioned above.

The time took by the study group to spend on social media networking have influenced on the particular individual's cortisol level. Moreover, that study group that uses less time on social media networking tend to have lower cortisol level or stays within the range and it's clear from the results obtained. $37.5 \%$ of the study group that uses less than one hour, their cortisol levels was normal in both morning and evening. Whereas another $37.5 \%$ that uses less than one hour has high cortisol concentration in the morning due to awakening and falls within the range by evening. Whereas for the study groups that use more than 2 hours, $97 \%$ tend to have high cortisol level in both the morning and evening. Thus this shows that the time used for accessing the social media sites has an impact towards the cortisol level. A recent study conducted on Facebook (FB) behaviors associated with diurnal cortisol in adolescent have stated that the greater the time spent on FB appears to be significantly related to higher psychological stress [8-11]. Moreover, from the analysis of cortisol concentration, the females tend to have higher cortisol level compare to males. A study was conducted on analysis of the perceived stress score (PSS) and blood pressure (BP) during examinations in medical students have stated that the female student tends to show more tendencies to be more stressed than the males [12]. High level of cortisol level seen in morning and evening indicates that the individual is undergoing stress. In a healthy person, the salivary cortisol level tends to follow the circadian rhythm where the cortisol level peaks in the early morning and decreases by evening. A study has stated that there is a 50- $100 \%$ increase in cortisol level which peaks about 30 minutes after awakening. If the response shows remarkable stability over weeks on the same individual, it indicates that the person is found to be in a prolonged stress exposure [13]. Thus, in this case, the study group shows a very high level of cortisol concentration in the morning, which can be associated with the usage of the social media networking site.

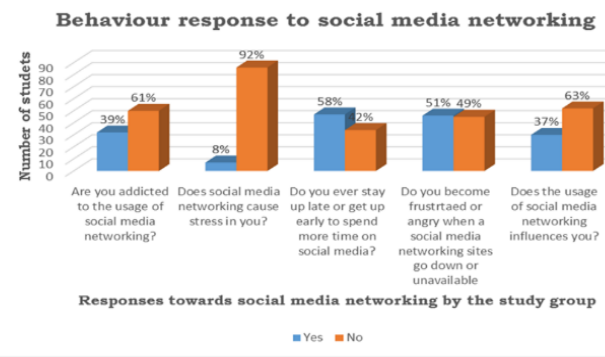

Figure 2: Behavior response to social media networking

Figure 2 shows the five questionnaires picked to study more on their social media networking behavior of the participant. From the first question, we can see that $39 \%$ of the participant agreed with the addiction to social media networking whereas the $61 \%$ of them have denied on the addiction to social media networking. The following question asked such as does the social media networking causes stress in you and shows $8 \%$ of the population have agreed that the usage of social media networking causes stress in them. However, the higher percentage of the participant have disagreed that the usage of social media networking does not cause stress in them. The third question that emphasis on their willingness to stay up late or get up early to spend more time on social media shows that $58 \%$ of them agreed that they wake up early and sleep late just to get access to social media networking, whereas $42 \%$ of them have denied. Furthermore, the following question on their frustration and anger when social media sites go down or unavailable shows $51 \%$ of them agreed and $49 \%$ denied. The last question was about the influences of social media networking states that $37 \%$ of them have agreed whereas $63 \%$ of them have denied on the statement.

As nowadays, social networking sites have become an alarm to many people in our society especially seen among the teenagers. This is because $58 \%$ of the study group wakes up early and sleeps late just to use the social media networking sites. Teenagers rely more on social media networking to meet new people, to communicate with friend and relatives, to upload pictures and many more, thus this is more relatable to the current lifestyle, where once they wake up they tend to find for their mobile to get access to the social media to update themselves. As they tend to practice it daily in their lifestyle. This gives an impact, to an individual because, when a person gets too addicted they are willing to sacrifice their sleep to achieve what they want. Besides that, the same goes when it comes to the night before they go to sleep, where it is a must to use a social media networking site before they go to sleep. Excess usage and addiction to social media networking site may disrupt a person's sleeping pattern. An individual needs a sufficient hour of sleep in order to sustain a healthy lifestyle and for proper metabolism of the body. Thus when a person lacks in their sleeping hour, this may also cause stress and eventually increase in cortisol level. Furthermore, when a person is too addicted to the usage of social media networking, they tend to get stress up easily if anything goes wrong while serving the net, for an example, if the social media networking goes down or unavailable for a while they tend to get frustrated and angry, which will also trigger the increase of stress level. This can be correlated with the questionnaire answered by the study group where $51 \%$ of them agree that they get frustrated and angry when the social media networking sites go down. This two usage of social media networking behavior has an impact on the cortisol level as it causes stress in the study group which is proven by the results obtained.

The study group was then divided into two types of the group based on the time spent on social media, which are the group 1 consist of less than the one-hour usage of social media, and group two for those study group that uses more than two hours of social media usage, as shown in Table 1. Those who use less social media networking site and spend less time on social media was kept as the control value to evaluate and compare the different cortisol level among the students who spend on social media networking site. From the questionnaire analyzed, a table was constructed based on the group divided according to time spent on social media networking as shown below.

Table 1: Total population

\begin{tabular}{|l|l|}
\hline Female & 53 \\
\hline Male & 13 \\
\hline Total population & 66 \\
\hline
\end{tabular}

From the table 1, the specific study group which involves both female $(n=53)$ and male $(n=13)$ was chosen based on the time spent on social media to estimate their cortisol level and to test whether there is an association with the usage of social media networking. This is because, from the time spent on social media networking can be correlated with the cortisol level and from this, a person stress level can be identified by correlating with the time spent on social media networking. A total population of 66 study group was chosen from the analyzed questionnaire to carry out the assay to determine the cortisol level.

\section{Analysis of stress using salivary cortisol}

A very high cortisol level seen among the study group in the morning as well as night. The cortisol level in the morning are seen to be high in males and females. There are fewer percentage of study group's cortisol level which is within the range or normal. Whereas in the night, the cortisol level seen in male and 
female seems to be high too and less percentage of study group's cortisol level falls within the range.

\section{Morning Cortisol level}

The figure 3 shows the morning cortisol concentration seen in male study group. The results show a high level of cortisol concentration among the male study group.

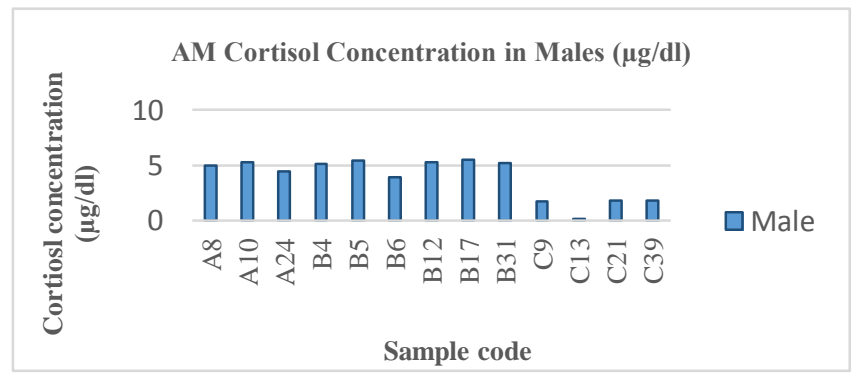

Figure 3: Morning cortisol concentration seen in male study group

The figure 4 shows the morning cortisol concentration seen in female study group. The results show a high level of cortisol concentration among the female study group.

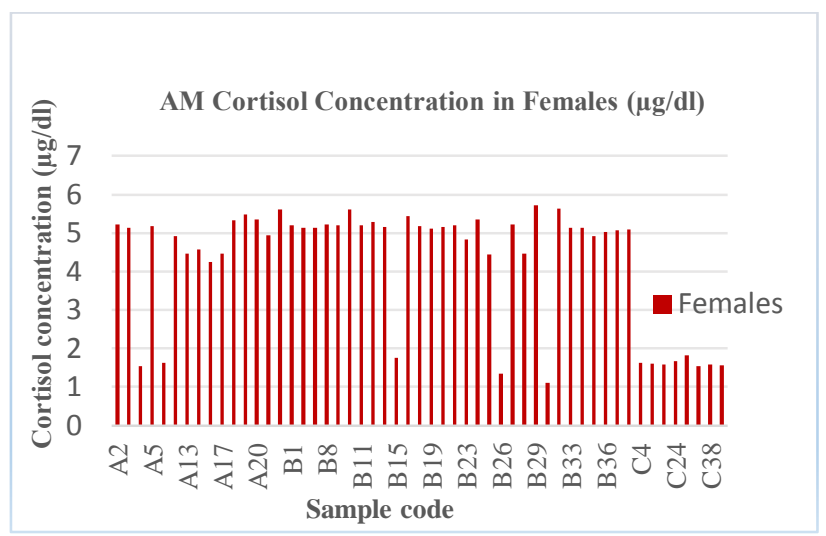

Figure 4: Morning cortisol concentration seen in female study group

The morning cortisol results show a high level of cortisol concentration in both male and female. The highest cortisol concentration seen was $5.72 \mu \mathrm{g} / \mathrm{dl}$ in female and $5.49 \mu \mathrm{g} / \mathrm{dl}$ in the male. Furthermore, the normal cortisol concentration was 1.34 $\mu \mathrm{g} / \mathrm{dl}, 1.11 \mu \mathrm{g} / \mathrm{dl}$ in females and $0.13 \mu \mathrm{g} / \mathrm{dl}$ in males. Whereas, the rest of the study group shows a high cortisol level ranging from (1.53- $5.6 \mu \mathrm{g} / \mathrm{dl})$.

\section{Evening cortisol level}

The figure 5. shows the evening cortisol concentration seen in male study group. The results show a high level of cortisol concentration among the male study group.

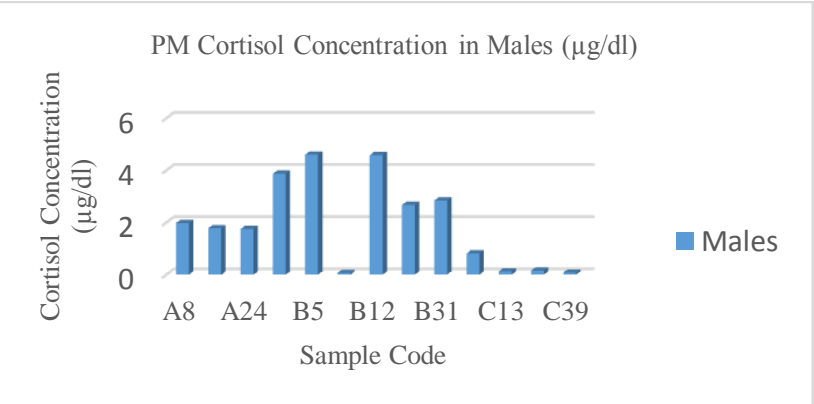

Figure 5: Evening cortisol concentration seen in male study group
The figure 6 shows the evening cortisol concentration seen in female study group. The results show a high level of cortisol concentration among the female study group.

PM Cortisol Concentration in Females $(\mu \mathrm{g} / \mathrm{dl})$

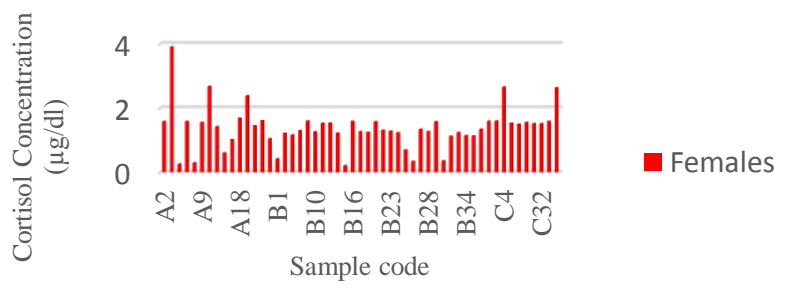

Figure 6: Evening cortisol concentration seen in female study group

The evening cortisol results show a high level of cortisol concentration in both male and female. The highest cortisol concentration seen was $3.9 \mu \mathrm{g} / \mathrm{dl}$ in female and $4.6 \mu \mathrm{g} / \mathrm{dl}$ in the male. Furthermore, the normal cortisol concentration was 0.21 $\mu \mathrm{g} / \mathrm{dl}, 0.26 \mu \mathrm{g} / \mathrm{dl}, 0.3 \mu \mathrm{g} / \mathrm{dl}, 0.34 \mu \mathrm{g} / \mathrm{dl}$ in females and $0.06 \mu \mathrm{g} / \mathrm{dl}$, $0.074 \mu \mathrm{g} / \mathrm{dl}, 0.12 \mu \mathrm{g} / \mathrm{dl}$ and $0.15 \mu \mathrm{g} / \mathrm{dl}$ in males. Whereas, the rest of the study group shows a high cortisol level ranging from $(0.81$ $4.58 \mu \mathrm{g} / \mathrm{dl}$ ).

Figure 7 shows the average value of cortisol concentration obtained in the morning seen in the female is higher than the male average cortisol concentration. The female average value shows $52 \%$ higher than the male average value.

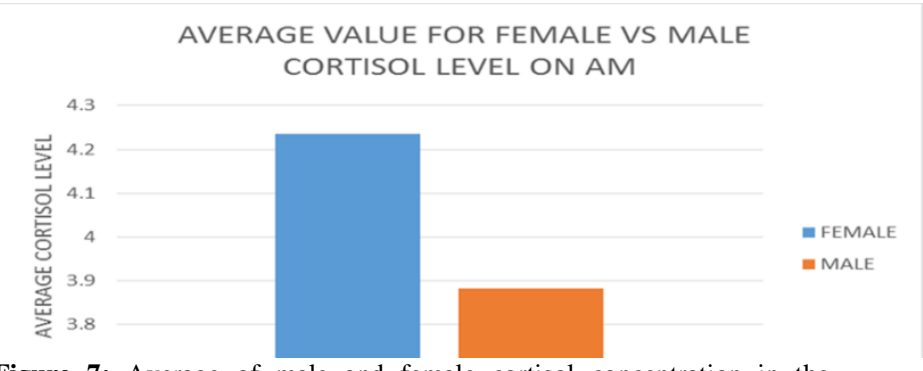

Figure 7: Average of male and female cortisol concentration in the evening $(\mu \mathrm{g} / \mathrm{dl})$

The figure 8 shows the average value of cortisol concentration obtained in the evening seen in the female is lower than the male average cortisol concentration. The male average value shows 58 $\%$ higher than the female average value.

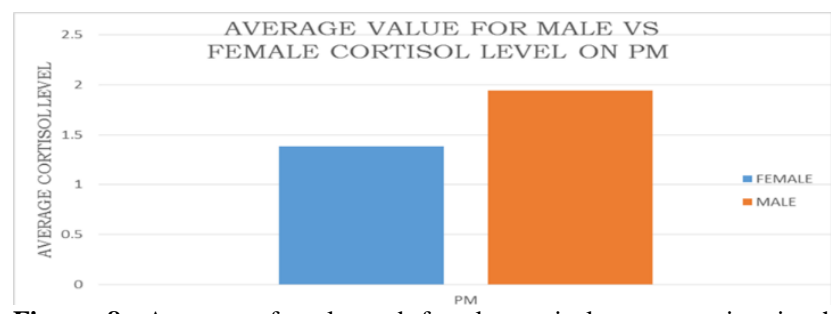

Figure 8: Average of male and female cortisol concentration in the evening $(\mu \mathrm{g} / \mathrm{dl})$.

A stress condition will elevate the cortisol level. When an individual is exposed to the stressor such as social media, this may give an impact and increase stress in that person in many ways, which will trigger the release of cortisol. The cortisol concentration of the study group and the usage of social media networking behavior shows there is an association, which can be proved by the results obtained by comparing the behaviors towards social media networking and cortisol level. Furthermore, the saliva sample collected twice a day to study the cortisol level among the study group and to identify whether there is a stress response due to the usage of social media networking. The morning saliva sample shows a high cortisol level. This is a 
natural physiological response by the body where the cortisol level peaks in the morning and reduces by evening. Although the cortisol level peaks in the morning, the results obtained by a test done shows that the cortisol level of the study group does not reduce to normal range by evening. However, even if there is the reduction in cortisol concentration, there were no far differences between the morning and evening concentration.

In summary, cortisol is a steroid hormone and also known as the stress hormone. Salivary cortisol plays a very important role in research and medical line as it has been routinely used as a biomarker to evaluate the stress level of an individual. Moreover, many studies have been conducted based on salivary cortisol to test the stress level by a stressor. Cortisol is said to be a stress hormone because, when a person encounters a stress response either psychologically or physiologically, the hypothalamus which is the part of the brain tends to get activated and releases a hormone known as corticotrophin releasing hormone (CRH) to the pituitary gland. Once it has arrived at the pituitary gland, it will then trigger the pituitary gland to secrete another hormone called as adrenocorticotropic hormone (ACTH) which will then send the signal to the adrenal gland to release cortisol to deal with the stressor. As we are in a technology-based era, social media networking has been playing a vital role in the society. The usage of social media networking has been the widely used application by everybody. Thus, in this present study, a preliminary study was carried out to determine the association of salivary cortisol and the usage of social media networking. From the study done, a positive association seen among the study's group cortisol level and the usage of social media networking behavior. High cortisol concentration was seen in both morning and evening in those study group that uses social media networking. A high cortisol concentration indicates that the specific student group is undergoing a chronic stress. This was proven by their time spent on social media and the usage of social media networking behavior. Whereas for those study group that uses less time on social media shows $67 \%$ have normal cortisol level. Thus, the study group that has a normal range of cortisol level was used to differentiate with the study group that has higher cortisol concentration. From the present study, it is very clear that there is an association of between salivary cortisol level and social networking behavior. It is more evident from the result obtained.

\section{Conclusion}

Results of the study suggest that students spending more time in social media networking have higher salivary cortisol level when compared to those students spending less time in social media Also, there exists a positive association between salivary cortisol level, a biomarker of stress and time spent on social media among MAHSA University students.

\section{Acknowledgement}

The authours would like to express thanks to MAHSA University and Dr. Chandramohan, Dr. Muhammed Jaseer and Dr. Paulraj Ponniah for their guidance, motivation immense knowledge, and support for making this manuscript.

\section{References}

[1] Selye H (1936), A Syndrome produced by Diverse Nocuous Agents, Nature 38,32.

[2] Haussmann MF, Vleck CM, Farrar ES (2007), A laboratory exercise to illustrate increased salivary cortisol in response to three stressful conditions using competitive ELISA, Advances in physiology education 31,110-5.

[3] Kalman BA, Grahn RE (2004), Measuring Salivary Cortisol in the Behavioral Neuroscience Laboratory, Journal of Undergraduate Neuroscience Education 2, A41-A9.
[4] Wilson RE, Gosling SD, Graham LT (2012), A Review of Facebook Research in the Social Sciences, Perspectives on Psychological Science 7, 203-20.

[5] D A. Cortisol - Its Role in Stress, Inflammation, and Indications for Diet Therapy. Today's Dietitian. 2009.

[6] [6] Hellhammer DH, Wust S, Kudielka BM (2009), Salivary cortisol as a biomarker in stress research, Psychoneuroendocrinology 34, 163-71.

[7] Selye H (1950), Diseases of adaptation, Wisconsin Medical Journal 49, 515-516.

[8] Keeffe GS, Clarke-Pearson K (2011), The Impact of Social Media on Children, Adolescents, and Families, Pediatrics,[10.1542/peds.2011-0054] 127,800.

[9] [9] Kontos EZ, Emmons KM, Puleo E, Viswanath K (2010), Communication inequalities and public health implications of adult social networking site use in the United States, Journal of health communication 15 Suppl 3, 216-35.

[10] Morin-Major JK, Marin MF, Durand N, Wan N, Juster RP, Lupien SJ (2016), Facebook behaviors associated with diurnal cortisol in adolescents: Is befriending stressful?, Psychoneuroendocrinology 63, 238-46.

[11] Pantic I, Damjanovic A, Todorovic J, Topalovic D, Bojovic-Jovic D, Ristic S. (2012), Association between online social networking and depression in high school students: behavioral physiology viewpoint, Psychiatria Danubina 24, 90-3.

[12] Ramesh Bhat M SM, Ganaraja B (2011), Eustress in Education: Analysis of the Perceived Stress Score (PSS) and Blood Pressure (BP) during Examinations in Medical Students, Journal of Clinical and Diagnostic Research [serial online]5, 1331-5.

[13] C K, DH H. Salivary cortisol. In: G II, editor. Encyclopedia of Stress v3. San Diego: Academic Press; 2000. p. 379-87. 